\title{
How the Brain Responds to the Destruction of Money
}

\author{
Cristina Becchio \\ University of Turin, Italy
}

\section{Uta Frith}

Åarhus University Hospital, Denmark, and

University College London, United Kingdom

\author{
Joshua Skewes and Torben E. Lund \\ Åarhus University Hospital, Denmark
}

\author{
Chris Frith \\ Åarhus University Hospital, Denmark and \\ Wellcome Trust Centre for Neuroimaging at \\ University College London, United Kingdom
}

\section{Andreas Roepstorff \\ Åarhus University Hospital, Denmark}

\begin{abstract}
Is our attitude to money like that to any other tool even though its use is symbolic and is not implemented in its physical structure? Knowledge of the functional use of concrete tools, such as hammers or screwdrivers, has been associated with activation of a left hemisphere network including the posterior temporal cortex, supramarginal gyrus, inferior parietal lobule, and lateral precuneus. Here we demonstrate that observing bank notes being cut up or torn, a critical violation of their function, elicits activation within the same temporo-parietal network. Moreover, this activation is the greater the higher the value of the banknote. This lends plausibility to a genuinely psychological interpretation of the explanation of money as a tool for parametrically symbolizing exchange.
\end{abstract}

Keywords: money, fMRI, social object, tool, functional use

In the same way that a literal tool such as a screwdriver satisfies our need to fix objects together and transcends the limited strength and dexterity of our hands, so money satisfies our need to exchange goods and transcends the limited capacity of our brains to keep track of and evaluate these exchanges (Mishkin, 1992). Arguably, this is the very purpose of money, based on the collectively agreed exchangeability between goods and services (Simmel, 1978). Lea and Webley (2006) call this the "tool" theory of money. The tool theory accepts the metaphorical extension of the idea of tool to money seeing money as means to an end: As a screwdriver is for screwing, money is for representing the value of goods and services, and it does this on a precise scale for tracking and evaluating their exchange.

For most tools, there is a strong link between function and physical structure: a screwdriver
Cristina Becchio, Department of Psychology, Center for Cogntive Science, University of Turin, Torino, Italy; Joshua Skewes, Section for Philosophy, MINDlab Åarhus University and Centre for Functionally Integrative Neuroscience, Åarhus University Hospital, Århus C, Denmark; Torben E. Lund, MINDLab Aarhus University and Centre for Functionally Integrative Neuroscience, Åarhus University Hospital, Århus C, Denmark; Uta Frith, MINDLab Åarhus University and Center for Functional Integrative Neuroscience, Århus University Hospital, Århus C, Denmark and Institute of Cognitive Neuroscience, University College London, London, United Kingdom; Chris Frith, MINDLab Åarhus University and Center for Functional Integrative Neuroscience, Århus University Hospital, Århus C, Denmark and Wellcome Trust Centre for Neuroimaging at University College London, London, United Kingdom; Andreas Roepstorff, Section for Anthropology, MINDLab Åarhus University and Center for Functional Integrative Neuroscience, Århus University Hospital, Århus C, Denmark.

This work benefited from financial support from the Danish National Research Foundation. The MRI scanner was donated by the John and Birthe Meyer Foundation. CB was supported by the Regione Piemonte, bando Scienze Umane e Sociali 2008, L.R. n. 4/2006.

Correspondence concerning this article should be addressed to Cristina Becchio, Center for Cogntive Science, Department of Psychology, University of Turin, Via Po 14, 10123 Torino, Italy. E-mail: cristina.becchio@unito.it 
has a physical structure that enables it to install and remove screws. This is not the case for money. In principle, any object can serve the function of money, from shells to gold, to paper, to binary code in bank computers. Money, for instance a 10-dollar bill, does not perform its function on the basis of its physical characteristics. None of the intrinsic properties of a 10dollar bill per se account for its function of being a medium of exchange for economic goods. Only our social practices tie this function to that medium. Put differently, what makes the piece of paper in my hand count as money is the fact that we, collectively, accept and recognize that the piece of paper has the status of money (Searle, 1995, 1998; Smith \& Searle, 2003). The status of money causes the piece of paper to have a value, which it does not have in virtue of its physical structure. This value is parametrically quantified by the numeral printed on the banknote: the higher the numeral, the greater the value symbolically represented by the banknote. However, neither the way the bank note represents the value of goods and services, nor the scale on which it represents them have any fixed relationship to the note's physical properties.

The fact that money is special in these ways raises an interesting set of questions. When we use money or see it being used, do we really employ similar representations as those of concrete tools such as screwdrivers? Is the tool explanation of money a genuinely psychological or only a metaphorical explanation? These questions can be addressed at the neurobiological level: do the brains of individual money users treat money as a tool?

Several neuroimaging studies have explored the neural representation of tools. A common finding is that observing tools compared with observing nonmanipulable objects such as houses or faces activates a distributed cortical network involving frontal, parietal and temporal areas within the left cerebral hemisphere (Johnson-Frey, 2004). Premotor frontal and posterior parietal activation is considered to describe tools in terms of their motor affordances (Jeannerod, Arbib, Sakata, \& Rizzolatti, 1995). In particular, premotor frontal regions are considered to encode graspable features (e.g., how to grasp and hold an object), associated with any graspable object that may involve a motor act. Parietal areas, including the inferior parietal lobule and the lateral precuneus, are believed to enable retrieval of tool-specific motor information (e.g., how to use a screwdriver; Vingerhoets, 2008). Posterior temporal areas are activated during semantic tasks involving tool naming and categorization and are therefore thought to be implicated in tool identification (Chao, Haxby, \& Martin, 1999).

Activation within the temporo-parietal network encoding critical knowledge of the functional use of tools has been demonstrated for familiar tools such as knives, fountain-pens, and nutcrackers (Vingerhoets, 2008). Here we investigated whether activation of a similar network is associated with knowledge of the function of money.

We asked Danish participants to view a series of videos in which different actions were performed on actual banknotes with a value of either of 100 Kroner (approximately 13 Euro/18 US Dollar) or 500 Kroner (approximately 67 Euro/91 US Dollar), or on valueless pieces of paper of the same size printed with a scrambled picture of these notes (Figure 1). We contrasted actions that were appropriate to money (folding or looking at valuable notes or valueless paper) and actions that were inappropriate (tearing or cutting notes or paper). Tearing and cutting destroy the value of the money and are thus violations of social norms. These actions negate the note's use as a tool and therefore directly interfere with its function. In contrast, folding a note or looking at it has no effect on its function and does not distinguish it from normal manipulation of a piece of paper. Because the socially agreed function of money is to represent value, we expected that these different actions would act as a strong stimulus for the network representing the functional use of money. Specifically, we expected to find increased activity in the tool network. Kellenbach, Brett, and Patterson (2003) observed that whereas premotor and posterior temporal activity is automatically elicited by the mere observation of familiar tools, parietal activity underling tool-use knowledge is selective for explicit retrieval of action information associated with tools. If the brain treats money as a tool, then causing participants to think about the functional use of money by violating this use should result in enhancement of activity in tool use areas. Furthermore, we predicted that the value of the banknote would elicit differential activation in functional use areas, because the function of money is the 

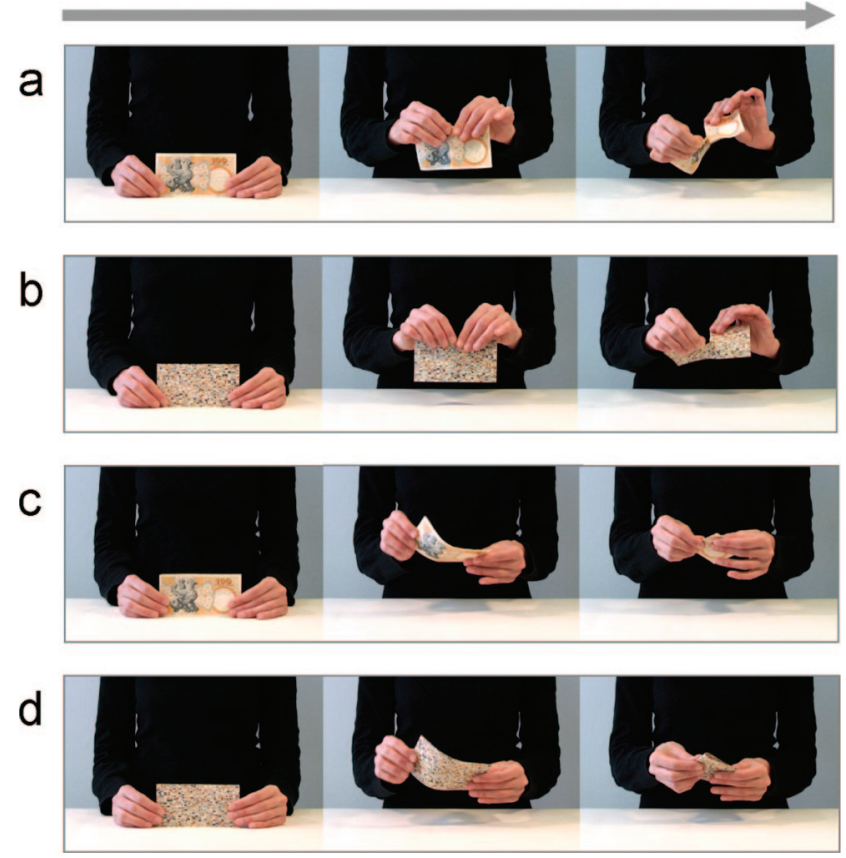

Figure 1. Single frames extracted from video clips representing a human actor performing (a) a destructive action on a banknote; (b) a destructive action on a printout of a scrambled banknote; (c) a nondestructive action on a banknote; (d) a nondestructive action on a printout of a scrambled banknote.

representation of a precise value. In particular, we predicted that the greater the value of the banknote the greater the activation within functional use areas, when participants observed the money being destroyed.

\section{Method}

\section{Participants}

Twenty adults (17 women, three men) with a mean age of 22 years (range $=20-29$ ) participated in a 1-hr testing session. All participants were right-handed, with no history of psychiatric or neurological disease. All participants were Danish and therefore suitably familiar with the currency presented in the stimulus set. The study was approved by the local research ethics committee and informed written consent was obtained prior to scanning.

\section{Stimuli}

During scanning, participants observed 128 video clips of $6.5 \mathrm{~s}$ duration, of which there were four types. Each video clip showed a pair of human hands acting on an object. In half the videos the object was a banknote. In the other half, the object was a printout of a scrambled banknote. The human actor could either perform a destructive (cutting or tearing) or nondestructive action (folding or looking at; Figure 1). Two different versions of each video kind were recorded. The videos were presented using Presentation (www.neuro-bs.com). The stimuli were projected onto a screen in the scanner room, which was viewed by the participants via a $45^{\circ}$ angled mirror mounted on the head coil (visual angle $15 \times 20$ degrees approximately). A test image was presented on the screen prior to scanning to ensure that the image was in focus and the subject could comfortably read the text presented.

\section{Design and Procedure}

Two videos of the same type were selected at random and without replacement to form 64 two-trial blocks of $13 \mathrm{~s}$, which were then presented in randomized order. Each block was 
preceded by instructions to pay attention to the action or the object in the videos. These instructions were presented for $3 \mathrm{~s}$. The experiment employed a $2 \times 2 \times 2$ factorial design, where factors were the action observed (destroy vs. nondestroy), the object observed (money vs. scrambled paper) and whether the instructions were to pay attention to the action or to the object. Sixteen videos were presented for each condition. Banknotes values were 100 Danish Kroner or 500 Danish Kroner. After each two-trial block, participants were asked either to indicate whether the action performed in the two videos was the same ("SAME ACTION?", e.g., YES if the action performed in two videos was folding; NO if the action performed in the first video was folding and the action performed in the second video was looking at), or to indicate whether the object in the two videos was the same ("SAME OBJECT?", e.g., YES if the object in the two videos was a 100 Kroner banknote; NO if the object in the first video was a 100 Kroner banknote and the object in the second video was a 500 Kroner banknote). The question asked always matched the attention condition instructed at the start of the block, and was answered by a button press within $3 \mathrm{~s}$. The correspondence of buttons to correct answers was randomized to control for motor effects associated with the response. Blocks were separated by a $1 \mathrm{~s}$ presentation of a centered fixation cross. After a practice session, each participant underwent a single experimental functional run lasting $23 \mathrm{~min}$ and $20 \mathrm{~s}$, during which they viewed all 64 two-trial blocks. The same-different judgment task ensured that participants directed their attention to the functionally appropriate/inappropriate manipulation of banknotes and scrambled pieces of paper.

After scanning, participants completed two questionnaires: a quantitative questionnaire using a visual analogue scale and a qualitative questionnaire using open ended questions. The quantitative questionnaire comprised comfort ratings (How comfortable did you feel?) and arousal ratings (How aroused did you feel?). Participants were presented with each video again and asked to rate it on a scale ranging from 1 to 9. Comfort and arousal were measured using self-assessment manikin (SAM; Bradley \& Lang, 1994). The qualitative questionnaire comprised 7 open ended questions and is displayed in Table 1. Questions 1-6 served
Table 1

Qualitative Post-Scanning Questionnaire

1. Describe what you saw in the videos. What did you think about it?

2. How did it make you feel?

3. In some of the videos you saw paper being cut or ripped. Have you ever done this? Would you do it?

4. In some of the videos you saw money being cut or ripped. Have you ever done this? Would you do it?

5. Did you notice any differences in the way the actions were performed?

6. Did you think that the money was real?

for exploratory purposes. Question 7 was an exclusion criterion for people not believing in the reality of the banknotes being manipulated.

\section{Scanning Procedure}

Scanning was performed using a GE Signa 3T Excite HDx with TwinSpeed gradients (40 $\mathrm{mT} / \mathrm{m}, 150 \mathrm{mT} / \mathrm{m} / \mathrm{ms}$ ) running software version 14M4. A gradient echo-planar (EPI) T2* weighted sequence sensitive to blood oxygenation level-dependent (BOLD) contrast was used to acquire 400 volumes each consisting of 39 slices. These $3.5 \mathrm{~mm}$ thick slices were acquired in interleaved order with a TR of $3500 \mathrm{~ms}$, TE of $30 \mathrm{~ms}$, a flip angle of $90^{\circ}$, a field of view of $240 \times 240 \mathrm{~mm}$, and a matrix size of $128 \times 128$. Prior to each functional run, five images were acquired and discarded to allow for longitudinal magnetization to approach equilibrium.

\section{Preprocessing of Functional Magnetic Resonance Imaging (fMRI) Data}

Data were preprocessed using SPM5 (Statistical Parametric Mapping, Wellcome Department of Imaging Neuroscience, University College London, London, United Kingdom; http:// www.fil.ion.ucl.ac.uk). Scans were realigned to the first volume (Friston et al., 1995), unwarped (Andersson, Hutton, Ashburner, Turner, \& Friston, 2001), slice-time corrected, spatially normalized to a standard template with a resampled voxel size of $2 \times 2 \times 2 \mathrm{~mm}$ (Ashburner, \& Friston, 1999), and smoothed using a Gaussian kernel with a full width at half maximum (FWHM) of $10 \mathrm{~mm}$. In addition, high-pass temporal filtering with a cut-off of $128 \mathrm{~s}$ was ap- 
plied to reduce low frequency noise associated with signal drift.

\section{Statistical Analysis for fMRI Data}

A random effects analysis was conducted on the data using SPM5. The variance in the BOLD signal was first decomposed in a first level model, with separate regressors coding for sustained activity in each of the 8 conditions within the experiment's factorial design and parametric modulations included for the value of the money presented in the videos. Individual within-subject models were in this way separately estimated and contrasts were defined for each experimental condition and for the effect of the parametric modulation.

Contrast images for each participant were then entered in separate analyses to test whether violations of money norms were associated with increased activity in the tool network, and to test whether this activity was parametrically modulated by the value of the money on which the actions were performed. In the first analysis, contrast images relating to experimental conditions were included with subject effects in a second level analysis of variance (ANOVA), which was corrected for violations of sphericity (Friston et al., 2002). The contrast for the main effect of the type of object presented was obtained for the whole brain. This produced an image, which was used as an inclusive mask for the contrast for the interaction between the actions performed and the objects acted on in the videos (Friston, Rotshtein, Geng, Sterzer, Henson, 2006). The statistical threshold for all contrasts was set to $p<.05$ (corrected for multiple comparisons using False Discovery Rate, FDR) and an extent voxel size of $k=20$ was consistently applied (Genovese, Lazar, Nichols, 2002).

In the second analysis, the first level contrasts for the effect of parametric modulation were entered into a one-sample $t$ test, second level random effects model. This analysis was masked inclusively with the regions where a significant interaction between action and object types was observed in the first analysis.

Anatomical structures were identified using a combination of the Talairach Atlas and overlaying the activation maps on the top of the spatially normalized, unsmoothed, mean EPI images averaged over subjects. This procedure accommodates the suboptimal spatial normalization of regions in the EPI images close to air-tissue boundaries.

\section{Results}

\section{Behavioral Data}

Accuracy for responding to whether the actions and objects were the same in the two videos ranged from $95 \%$ to $99 \%(M=97 \%$, $S D=1.15 \%)$. Response time ranged from 935 $\mathrm{ms}$ to $988 \mathrm{~ms}(M=963 \mathrm{~ms}, S D=173 \mathrm{~ms})$. A repeated measures ANOVA with three withinsubject factors, observed action (destroy vs. nondestroy), observed object (money vs. scrambled paper) and attention (attention paid to the action vs. attention paid to the object) was applied to response accuracy and revealed no significant effects. An ANOVA on response time also revealed no significant effects.

Following the scanning session we assessed participants' level of comfort and arousal during the observation of destroying and nondestroying action performed either on banknotes or scrambled papers.

\section{Comfort Ratings}

A repeated measures ANOVA with the two within-subject factors, observed action (destroy vs. nondestroy) and observed object (money vs. scrambled paper) revealed a significant interaction effect, $F(1,19)=20.280, p<.001$. Post hoc contrasts (Bonferroni corrected) confirmed that participants felt less comfortable during observation of destroying actions performed on money than during observation of destroying action performed on scrambled papers $(p<$ .001). By contrast, no significant difference in comfort level was revealed when comparing the observation of nondestroy action performed on money and scrambled papers $(p>.05)$.

\section{Arousal Ratings}

A repeated-measures ANOVA on arousal ratings with two within-subject factors, observed action (destroy vs. nondestroy) and observed object (money vs. scrambled paper) also yielded a significant interaction effect, $F(1$, $19)=10.710, p<.005$. Post hoc contrasts (Bonferroni corrected) showed that participants 
felt more aroused during observation of destroying actions performed on money than during observation of destroying action performed on scrambled papers $(p<.001)$. Furthermore, arousal was greater when observing nondestroying actions performed on money compared with nondestroy actions performed on scrambled papers $(p<.001)$.

All participants reported believing in the reality of the banknotes being manipulated.

\section{fMRI Data}

To test whether violations of money norms were associated with increased activity in the tool network fMRI data were subjected to a repeated-measures analysis of variance (ANOVA). Observed action (destroy vs. nondestroy), observed object (money vs. scrambled paper) and attention (attention paid to the action vs. attention paid to the object) were treated as within-subjects factors. Peak activity and stereotaxic coordinates for activations are listed in Table 2.

\section{Main Effect of Action}

Observing cutting and tearing action relative to folding and looking at actions (destroy $>$ non destroy) increased activity bilaterally in a num-

Table 2

Activations Related to Main Effects and Interaction

\begin{tabular}{|c|c|c|c|c|c|c|}
\hline Anatomical regions & Brodmann areas & \multicolumn{3}{|c|}{ Coordinates $(x, y, z)$} & $Z$ & Cluster size \\
\hline \multicolumn{7}{|l|}{ Main effect of action } \\
\hline R Precuneus & 7 & 26 & -54 & 60 & $\inf$ & 36,944 \\
\hline L Precuneus & 7 & -28 & -54 & 62 & inf & \\
\hline Claustrum & & -38 & -6 & 6 & 5.32 & 241 \\
\hline R Superior temporal gyrus & 22 & 66 & -28 & 4 & 5.29 & 861 \\
\hline R Precentral gyrus & 6 & 26 & -10 & 58 & 4.65 & 292 \\
\hline L Precentral gyrus & 6 & -22 & -10 & 60 & 4.11 & 192 \\
\hline \multirow{2}{*}{ L Cerebellum posterior lobe } & & -4 & -78 & -34 & 3.76 & 131 \\
\hline & & 12 & -76 & -50 & 3.60 & 116 \\
\hline \multicolumn{7}{|l|}{ Main effect of object } \\
\hline L Inferior occipital gyrus/fusiform & $18 / 19$ & -32 & -88 & -12 & $\inf$ & 7,735 \\
\hline R Middle occipital gyrus & 18 & 40 & -84 & -6 & inf & 16,247 \\
\hline L Superior parietal lobule & 7 & -34 & -48 & 66 & 4.35 & 3,124 \\
\hline R Middle frontal gyrus & 8 & 46 & 36 & 40 & 4.04 & 448 \\
\hline L Middle frontal gyrus & 10 & -36 & 58 & -10 & 3.61 & 371 \\
\hline R Middle frontal gyrus & 11 & 44 & 52 & -18 & 3.56 & 199 \\
\hline R Precentral gyrus & 6 & 60 & 4 & 28 & 3.88 & 239 \\
\hline L Posterior cingulate cortex & 31 & -16 & -24 & 36 & 3.85 & 144 \\
\hline L Middle frontal gyrus & 10 & -36 & 58 & -10 & 3.61 & 371 \\
\hline R Middle frontal gyrus & 11 & 44 & 52 & -18 & 3.56 & 199 \\
\hline L Medial frontal gyrus & 10 & -16 & 56 & -18 & 3.30 & 104 \\
\hline \multicolumn{7}{|l|}{ Main effect of attention } \\
\hline L Inferior temporal gyrus & 37 & -54 & -72 & 2 & 5.37 & 1,505 \\
\hline L Inferior parietal lobule & 40 & -66 & -38 & 40 & 4.94 & \\
\hline L Parahippocampal gyrus & 28 & -20 & -16 & -20 & 4.75 & 222 \\
\hline R Middle temporal gyrus & 39 & 44 & -58 & 6 & 4.69 & 599 \\
\hline L Lingual gyrus & 19 & -16 & -60 & 4 & 4.08 & 163 \\
\hline L Parahippocampal gyrus & 30 & 8 & -52 & 2 & 3.82 & 174 \\
\hline \multicolumn{7}{|l|}{ Interaction action by object } \\
\hline L Fusiform gyrus & 19 & -32 & -74 & -18 & 4.68 & 1,389 \\
\hline L Inferior temporal gyrus & 37 & -26 & -66 & -10 & 4.41 & \\
\hline L Lingual gyrus & 18 & -28 & -76 & -8 & 4.26 & \\
\hline L Precuneus & 31 & -28 & -76 & 28 & 3.58 & 111 \\
\hline L Cuneus & 31 & -24 & -78 & 38 & 3.39 & \\
\hline
\end{tabular}

Note. Anatomical and Brodmann areas (BA) and Montreal Neurological Institute (MNI) coordinates of the maxima within each cluster. All the reported effects are significant at $p<.05$ False Discovery Rate (FDR) corrected at voxel level. Extent voxel size $k=100$. 
ber of regions, including the precuneus (Brodmann area 7), the posterior portion of superior temporal gyrus (BA 22) and the precentral gyrus (BA 6). Within the right hemisphere, activation extended medially to include the parahippocampal gyrus (BA 35) and the posterior cingulated cortex (BA 31). As for the activations the left hemisphere, a peak was located within the superior temporal pole (BA 38). An additional cluster of increased activity was revealed within the frontal cortex and encompassed the inferior frontal gyrus (BA 47) and the middle frontal gyrus (BA 11).

\section{Main Effect of Observed Object}

For the main effect of observed object (money $>$ scrambled paper) a large area of bilateral activation was observed in the occipital-temporal cortex (BA 18/19). Additional loci of increased activity were found bilaterally in the superior temporal pole, in the lateral aspects of the prefrontal cortex (BA 6 and BA 9) and in the ventral prefrontal cortex at the level of the middle frontal gyrus (BA 10/11). Within the left hemisphere, differential activations were observed in the precuneus (BA 7), in the adjacent posterior cingulated cortex (BA 31), in the supramarginal gyrus (BA 40) and in the superior parietal lobule (BA 7). Finally, a peak of activation was observed in the right superior temporal sulcus (BA 22/42).

\section{Main Effect of Attention}

Significant activations related to the main effect of attention (attention paid to the action $>$ attention paid to the object) were revealed within left occipito-temporal regions. Activation encompassed the inferior temporal gyrus
(BA 37) and the lingual gyrus (BA 19) and extended anteriorly the parahippocampal gyrus (BA 28/30). Additional loci of increased activity were found within the left inferior parietal lobule (BA 40), including the temporo-parietal junction (BA 39), the right middle temporal gyrus (BA 39) and the right precuneus (BA 31).

\section{Interaction Observed Action by Observed Object}

The repeated measures ANOVA revealed a significant interaction between action and object types [(destroy money-destroy scramble paper) - (non destroy money-non destroy scrambled paper)] within the left posterior temporal cortex, including fusiform gyrus (BA 19/ 37 ) and the left posterior precuneus (BA 31; Figure 2).

\section{Parametric Modulation for the Value of Money Presented}

To test whether the main effect of action increased as a function of the value of moneythe greater the value of the banknote, the greater the activation within functional use areas - an additional second level analysis was performed including parametric modulations for the value of money presented in the videos. Parametric modulation for the value of money presented was revealed within the fusiform gyrus as well in the left posterior precuneus (Figure 3).

\section{Discussion}

We investigated whether brain circuitry identified as relevant for representing functional tool knowledge also provides a basis for the repre-
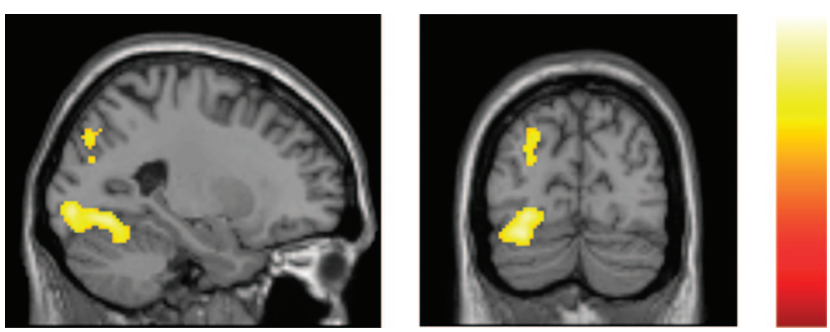

Figure 2. Left posterior temporal cortex (fusiform gyrus) and left posterior precuneus activation emerged from the interaction between action and object types. 


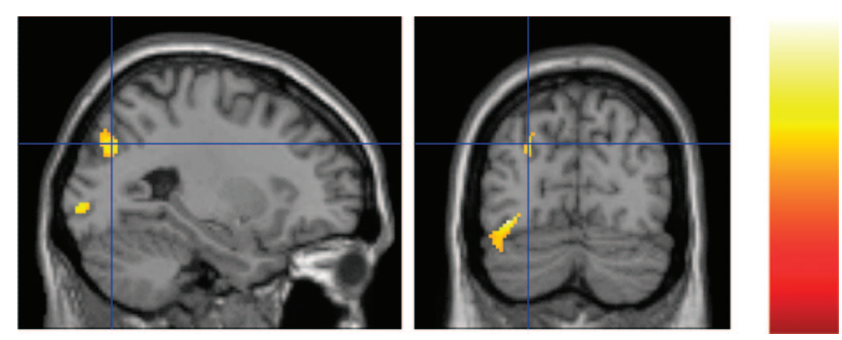

Figure 3. Activation within the left posterior temporal cortex and left posterior precuneus was parametrically modulated by the value of the money presented.

sentation of the functional use of money. We contrasted actions that were appropriate to money (folding or looking at valuable notes or valueless paper) and actions that were inappropriate (tearing or cutting notes or paper) as they negate the note's use as a tool and therefore directly interfere with its function.

Behavioral assessment after scanning confirmed the saliency of the manipulation: participants felt more aroused and less comfortable during observation of destroying actions performed on money than during observation of destroying action performed on paper.

At the neural level, a significant effect of interaction between action and object types was observed within the left fusiform gyrus and the left posterior precuneus; regions within the same left-lateralized temporo-parietal network associated with functional tool use.

Fusiform gyrus activity has been linked to tool identification (Johnson-Frey, 2004). Visual processing of tools has been associated with activity in the medial fusiform gyrus, which has been shown to respond more to tools than to animals and to be sensitive to different object categories (Chao et al., 1999). Because the fusiform gyrus is part of the ventral object-vision pathway, it is possible that a general function of this region is to represent visual features shared by members of an object category. The medial fusiform activity identified in the present study is therefore likely to be involved in activation of stored information about the sensory features that identify money as an object categorically distinct from visually similar scrambled banknotes. That fusiform activity was modulated by the value of the money presented supports this interpretation, and suggests that the categorical representations in this region are fine- grained enough to distinguish between banknotes of different value.

Precuneus activity has been linked to tasks involving visuospatial imagery and episodic memory (Cavanna \& Trimble, 2006). Recent studies suggest a functional dissociation within the precuneus between an anterior region associated with spatial imagery, and a posterior region associated with successful retrieval of memorized motor patterns (Cavanna \& Trimble, 2006; Knauff, Fangmeier, Ruff, Johnson-Laird, 2003; Ghaem et al., 1997). Vingerhoets (2008) reported the left posterior precuneus to be more active during the presentation of familiar tools compared with tools for which observers have little or no functional knowledge. However, the specific role of the precuneus in this task remains an open question. The present findings offer an answer. Because the function of money is not implemented in its physical structure, it does not provide any physical cues to its function, which must therefore be reconstructed from memory. It might therefore be hypothesized that within the larger left lateralized tool network, the posterior precuneus provides a memory-based representation of functionally appropriate tool use. That precuneus activity is parametrically modulated by the value of the money presented supports this interpretation, and suggests that value is one of the critical functional properties of money represented in this region.

No effect of interaction between action and object types was observed within inferior parietal lobule. Inferior parietal lobule has been proposed to store knowledge about the limb and hand positions necessary for the functional use of familiar tools (Buxbaum, Kyle, Tang, \& Detre, 2006; Vingerhoets, 2008). Because destroy- 
ing actions did not interfere with this specific aspect of functional use knowledge, this might indeed explain why interaction between action and object types failed to show any inferior parietal activation. It is also possible that toolhand position coupling is relevant only for tools the use of which requires the acquisition of specific motor skills. Because the use of money does not involve complex object-associated actions (except perhaps for professionals such as bank clerks), no differential activation within the inferior parietal lobule is observed when the manipulation of money is compared the manipulation of scrambled pieces of paper. Functional neuroimaging studies could be developed to discriminate between these two alternatives.

A possible confound that might affect this interpretation of the results is represented by the affective saliency of the destruction of money. Could it be that the reported effects are mediated by the manipulation of the level of comfort and arousal? Put differently, the destruction of money might have been associated with increased activity in the tool network, not because money was treated as a tool, but because the manipulation of money was more arousing? The same issue applies to parametric modulation for the value of money presented. Activation within functional use areas might be greater because, as the value of money presented increases, the participants' level of arousal and discomfort increase as well.

Although it is plausible that affective saliency modulate response to money destruction, a strong argument against this explanation of the present result is that we found no effect of money destruction within brain regions typically associated with monetary loss, including striatal areas and mesial frontal areas (e.g., Knutson, Westdorp, Kaiser, \& Hommer, 2000) or loss aversion, including the amygdala (e.g., De Martino, Camerer, \& Adolphs, 2010). Furthermore, we found no linear relation between brain activity and arousal and comfort ratings. After multiple comparisons over the whole brain (FDR), no significant activations were found, suggesting that increased activity in the tool network did not reflect affective reaction to money destruction.

\section{Conclusions}

Violation of social norms associated with money activates a network associated with tool use, and this network is parametrically modulated by the value of the money presented. This lends plausibility to a genuinely psychological interpretation of the explanation of money as a tool for parametrically symbolizing exchange. While research in economic psychology is uncovering some phenomena that this explanation cannot account forphenomena that suggest that money can also act as a drug (Lea \& Webley, 2006) - the fact that the brain does treat money as a tool for tracking exchange on a precise scale suggests that a tool explanation of money is more than just a useful metaphor.

\section{References}

Andersson, J. L. R., Hutton, C., Ashburner, J., Turner, R., \& Friston, K. J. (2001). Modeling geometric deformations in EPI time series. Neuroimage, 13, 903-919.

Ashburner, J., \& Friston, K. J. (1999). Nonlinear spatial normalization using basis functions. $\mathrm{Hu}$ man Brain Mapping, 7, 254-266.

Bradley, M. M., \& Lang, P. J. (1994). Measuring emotion: The self-assessment manikin and the semantic differential. Journal of Behavioral Therapy and Experimental Psychiatry, 25, 49-59.

Buxbaum, L. J., Kyle, K. M., Tang, K., \& Detre, J. A. (2006). Neural substrates of knowledge of hand postures for object grasping and functional object use: Evidence from fMRI. Brain Research, 1117, 175-185.

Cavanna, A. E., \& Trimble, M. R. (2006). The precuneus: A review of its functional anatomy and behavioural correlates. Brain, 129, 564-583.

Chao, L. L., Haxby, J. V., \& Martin, A. (1999). Attribute-based neural substrates in posterior temporal cortex for perceiving and knowing about objects. Nature Neuroscience, 2, 913-919.

De Martino, B., Camerer, C. F., \& Adolphs, R. (2010). Amygdala damage eliminates monetary loss aversion. Proceedings of the National Academy of Sciences, USA, 107, 3788-3792.

Friston, K. J., Holmes, A. P., Worsley, K. J., Poline, J.-B., Frith, C. D., \& Frackowiak, R. S. J. (1995). Statistical Parametric Maps in functional imaging: A general linear approach. Human Brain Mapping, 2, 189-210.

Friston, K. J., Penny, W., Phillips, C., Kiebel, S., Hinton, G., \& Ashburner, J. (2002). Classical and Bayesian inference in neuroimaging: Theory. Neuroimage, 16, 465-483.

Friston, K. J., Rotshtein, P., Geng, J. J., Sterzer, P., \& Henson, R. N. (2006). A critique of functional localizers. Neuroimage, 30, 1077-1087. 
Genovese, C. R., Lazar, N. A., \& Nichols, T. (2002). Thresholding of statistical maps in functional neuroimaging using the false discovery rate. Neuroimage, 15, 870-878.

Ghaem, O., Mellet, E., Crivello, F., Tzourio, N., Mazoyer, B., Berthoz, A., \& Denis, M. (1997). Mental navigation along memorized routes activates the hippocampus, precuneus, and insula. Neuroreport, 8, 739-744.

Jeannerod, M., Arbib, M. A., Rizzolatti, G., \& Sakata, H. (1995). Grasping objects: The cortical mechanisms of visuomotor transformation. Trends in Neurosciences, 18, 314-320.

Johnson-Frey, S. H. (2004). The neural bases of complex tool use in humans. Trends in Cognitive Science, 8, 71-78.

Kellenbach, M. L., Brett, M., \& Patterson, K. (2003). Actions speak louder than functions: The importance of manipulability and action in tool representation. Journal of Cognitive Neuroscience, 15, $30-46$.

Knauff, M., Fangmeier, T., Ruff, C. C., \& JohnsonLaird, P. N. (2003). Reasoning models and images: Behavioral measures and cortical activity. Journal of Cognitive Neuroscience, 15, 559-573.
Knutson, B., Westdorp, A., Kaiser, E., \& Hommer, D. (2000). fMRI visualization of brain activity during a monetary incentive delay task. NeuroImage, 12, 20-27.

Lea, S. E. G., \& Webley, P. (2006). Money as tool, money as drug: The biological psychology of a strong incentive. Behavioral and Brain Sciences, 29, 161-209.

Mishkin, F. S. (1992). The economics of money, banking and financial markets. New York: Harper Collins.

Searle, J. R. (1995). The construction of social reality. New York: Free Press.

Searle, J. R. (1998). Mind, language and society: Philosophy in the real world. New York: Basic Books.

Simmel, G. (1978). The philosophy of money. London: Routledge and Kegan Paul.

Smith, B., \& Searle, J. R. (2003). The construction of social reality: An exchange. The American Journal of Economics and Sociology, 62, 285-309.

Vingerhoets, G. (2008). Knowing about tools: Neural correlates of tool familiarity and experience. $\mathrm{Neu}$ roimage, 40, 1380-1391. 